\section{So wird die Fremdanamnese nach Nr. 4 GOÄ ehoben}

nen Terminen behandelt. Dann kann für jede Krankheit, wegen der es zu einer Fremdanamnese bzw. einer Unterweisung kommt, einmal die Nr. 4 angesetzt werden.

\section{MMW-KOMMENTAR}

Die Nr. 4 kann in derselben Sitzung auch neben der Nr. 1 berechnet werden. Ausnahme: Sämtliche Bestandteile der Legenden beider Nrn. richten sich an dieselbe Person, etwa wenn der Arzt mit den Eltern eines Kleinkinds

Tab. 1 Abrechnungsbeispiel: Besuch bei einer wegen Demenz kommunikationsgestörten Patientin

\begin{tabular}{|l|l|l|}
\hline GOÄ & Legende & \multicolumn{2}{|c|}{ Euro (2,3-fach) } \\
\hline 50 & Hausbesuch & 42,90 \\
\hline 7 & Wegegeld: 2 Kilometer & 3,58 \\
\hline 800 & Untersuchung Thorax & 21,46 \\
\hline 4 & $\begin{array}{l}\text { Neurologische Untersuchung } \\
\text { Fremdanamnese: } \\
\text { umfangreiche Befragung } \\
\text { der Pflegerin }\end{array}$ & 26,15 \\
\hline
\end{tabular}

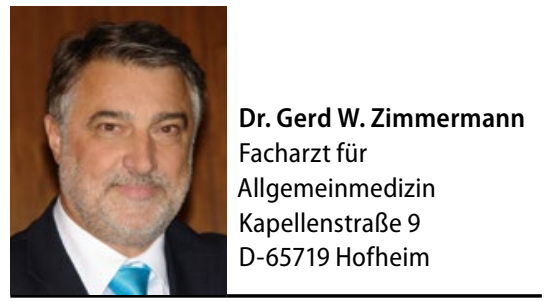

oder dem Betreuer eines kommunikationsgestörten Patienten spricht. Das haben 1996 der GOÄ-Ausschuss der Bundesärztekammer und 2001 das Landgericht Karlsruhe klargestellt (Az.: 1 S 90/99).

Auch neben der Nr. 100 ist die Nr. 4 nicht ausgeschlossen. Sie kann also angesetzt werden, wenn zur Klärung des Todes eines Patienten eine Fremdanamnese erhoben werden muss. Konkrete Krankheitsbilder werden in der Legende der Nr. 4 nicht genannt. Auf Basis einer recht eindeutigen Rechtsprechung erkennen die Versicherungsträger in der Regel aber nur krankheitsbedingte Kommunikationsstörungen an. Eine solche liegt nicht nur bei Kindern, behinderten Personen oder Toten vor, sondern z. B. auch bei Demenzpatienten. Daher kann der Besuch eines dementen Patienten zumindest einmal im Monat mit der Nr. 4 kombiniert werden.

Nicht ausreichend für die Berechnung sind kurze Anweisungen zur Krankenbehandlung oder kurze Befragungen von Bezugspersonen. Es bedarf einer umfangreichen, grundsätzlichen Befragung oder Unterweisung einer Bezugsperson. Aktuell wichtig: Auch wenn die Bezugsperson ein Dolmetscher ist, kann die Nr. 4 berechnet werden.

\title{
Eigenblutbehandlung rechtssicher abrechnen
}

Individuelle Gesundheitsleistungen (IGeL) sind etwas in Verruf geraten, man denke nur an den IGeL-Monitor der Krankenkassen. Die Eigenblutbehandlung wird hier negativ dargestellt, allerdings ist die Beurteilung der Eigenblutbehandlung auf die Behandlung von Tendopathien beschränkt. Die als Indikation wesentlich häufigere Immunstimulation wird nicht erwähnt. Grund- sätzlich kann aber von einer anerkannten Heilmethode ausgegangen werden, da die Behandlungsmethode in der GOÄ mit der Nr. 284 vertreten ist.

\section{MMW-KOMMENTAR}

Die Eigenblutbehandlung ist keine Kassenleistung und wird deshalb bei GKV-Versicherten nach vorherigem Abschluss eines Behandlungsvertrages nach der Nr. 284 liqui- diert (90 Punkte, 12,07 Euro bei 2,3-fachem Satz). Die Nr. schließt die Blutentnahme und die intramuskuläre Reinjektion ein. Wird das entnommene Blut vor der Reinjektion besonders behandelt, kann mit entsprechender Begründung ein höherer Multiplikator angesetzt werden. Auch ggf. notwendige Beratungen oder Untersuchungen werden gesondert berechnet, z. B. nach Nr. 1 und Nr. 5 (jeweils 10,72 Euro bei 2,3-fachem Satz). 